\title{
Dynamic symbol for electronic map, network map and the ability to create dynamic symbol on the map with the online answering machine
}

\author{
Vinh Tuyet Thi Tran*
}

Faculty of Geomatics and Land Administration, Hanoi University of Mining and Geology, Vietnam

\begin{tabular}{l} 
ARTICLE INFO \\
\hline Article history: \\
Received 13th Feb. 2020 \\
Revised 20 $0^{\text {th }}$ May 2020 \\
Accepted 30th June 2020
\end{tabular}

Keywords:

Dynamic map,

Dynamic symbol,

Map GIFs,

Online answer engine,

Wolfram Alpha.

\section{ABSTRACT}

In visualization map, innovation is always needed to make geographic data more accessible to everyone. As well as other sciences, it must be based on solid theory. Currently, some electronic maps and network maps have used dynamic symbols but to summarize this new form of map symbols in Vietnam, there are no specific documents. This article provides some descriptive and applied basis for forming dynamic symbols for electronics maps, network maps. Along with that, the article introduces the Wolfram Alpha online answering engineand the way to communicate with this artificial intelligence engine to create dynamic symbols on the map showing the development of the outbreak Covid -19. Initially, this result opened up the possibility of using a new tool for map makers.

Copyright @ 2020 Hanoi University of Mining and Geology. All rights reserved.

\section{Introduction}

The electronic map displayed on mobile phones, computers, television, and navigation devices have been used in Vietnam in recent years. That opens up many trends for showing the map with animation images, or "dynamic symbol." Compared with static symbols, standing still on the map, the dynamic symbols create more focus and bring more efficiency to the map presentation (Lai Poh Chin, 2004). In Vietnam, the map with dynamic symbols mainly appears on the

${ }^{*}$ Corresponding author

E-mail: tranthituyetvinh@humg.edu.vn

DOI: 10.46326/JMES.2020.61(3).10 weather forecast program of television and navigation equipment for for means of transport (Bui Ngoc Quy, 2019). The reason for the meager appearance of dynamic-use maps is because of the effort and time required to create the map. In foreign countries, this form of map has been conducted by Lai Poh Chin to conduct experiments on human cognitive ability with dynamic symbols (Lai Poh Chin, 2004). The popular used this type of symbols in television weather forecasting programs has been seen in the BBC since 2011 (Fairbairn David, 2013). By 2014, Paweł Cybulski experimented with creating dynamic symbolic maps using Adobe. Flash to make rotable symbols (Paweł Cybulski, 2014). And now, websites, electronic lectures, even 
applications that record the travel logs of social networking sites all see dynamic symbols. This article summarizes the basic principles of changing the motion when using dynamic symbols and introduces the ability to create dynamic symbol maps using Wolfram Alpha online answering engine. This is a way to create simple dynamic symbol maps that have appeared in recent years in the world but not much is known in Vietnam. The applications of Wolfram Alpha in Vietnam are mainly used to calculate mathematical formulas in high school mathematics programs and function graphs in teaching and learning. The task of this study is to experiment with drawing dynamic symbols in maps using the Wolfram Alpha tool.

\section{Theoretical basis of dynamic symbol on map and application method}

\subsection{Theoretical basis of dynamic symbol on map}

Map symbols are divided into 3 types: point symbol, line symbol, and area symbol. These types of symbols have two ways of representing a twodimensional (2D) or a three-dimensional (3D) (Mai Van Sy, 2019). The ways shown above are static with the whole map space. But the map must always guide the viewer to know the information of the map respectively from the main to the secondary through the map's symbol system. To do this, all the abovementioned symbols must work together to indicate which symbols are the important ones in the map (map grammar). The content of these important symbols needs to be identified first, the content of the less important symbols are identified later. To highlight the important symbols, 6 variable values of the symbol are clarified, which are shape, size, direction, internal structure, brightness, and color changes, to create focus on the preferred symbol. (Tran Trung Hong, 2001). In order for a map to create a more focused viewer with a number of symbols that need to be identified first (often called a thematic symbol layer), these symbols will have a more realistic appearance, large sizes, in the opposite direction to other symbols (often called geographic base symbol layer). These thematic symbols need a simple internal structure, brighter or darker or colourful than others. It will make us look at and identify their content before other symbol (Tran Trung Hong, 2001). These variables, if they are flexible application with the map notation system, not only represent the content in turn by layer of information (thematic information layer and geographic base information layer), do not overlap, but also bring the maximum amount of information on the map.

Currently, map is often displayed on electronic screens. The application of animation images with map symbols is positive (Kim Quang Minh, 2019). In addition, the human eye nerve as well as those of mammals are more capable of tracking moving images than the still ones. In other words, on the background of the stationary scene, people focus on moving points rather than on stationary points. Also, if the map uses both static symbols and dynamic symbols, it will increase the attraction to dynamic symbols. Therefore, the map can arrange many symbols but still makes the viewer look focused, without missing information. (P. Peterson Michael, 1993).

Technically, point symbols, line symbols, and area symbols can applied motion. These three types of symbols cannot change their relative position on the map, hence, any element that is allowed to move is the index that needs to be specified. Point symbols and line symbols can be created animation at the valence as shape (Figure 1), size (Figure 2), brightness (Figure 3), direction (Figure 4), internal structure (Figure 5) and colors (Figure 6). Area symbols often create animation in brightness, internal structure, and color. Symbols that uses motion based on a type of animation is naturally less attractive than symbols use many type of animation, but the number of symbols on the map is often large, therefore, it is usually used only with a type of animation to avoid chaos. (Qian Cheng, 2013).

Changing brightness, internal structure, color will not affect the area occupied by symbols on the map. Most other animations of symbol have a change occupied area. Usually, in experimental applications, it is shown that the ability to identify the symbols best with a change in area is about 1.5 times the original area (DiBiase David, 1992). 

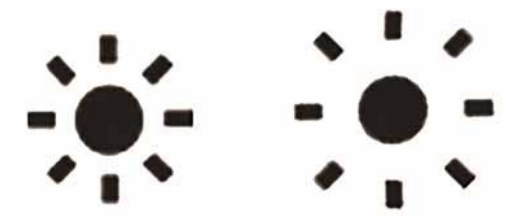

b. Scene 2

c. Scene 3

a. Scene 1

Figure 1. Dynamic symbol change shape.
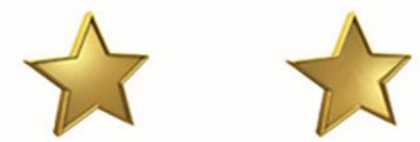

b. Scene 2

c. Scene 3

a. Scene 1

Figure 3. Dynamic symbol change brightness.
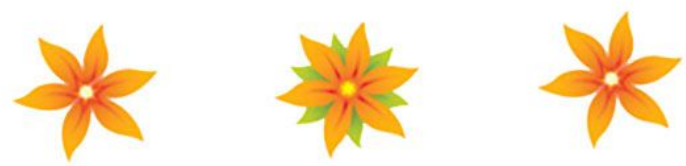

\section{a. Scene 1 \\ b. Scene 2 \\ c. Scene 3}

Figure 5. Dynamic symbol change internal structure.

If all symbols on map have animation then that will disturb the sight. Only some symbols, that be recognized in advance, need create animation. The same type of symbols, only used the animation for the symbols are in important position on the map and the symbols in other positions on the map can stand still (Figure 7).

We also need to abide by the rules of the map. Therefore, with one type of symbol, only one type of motion should be created.

In order to identify the animation of the symbol clearly, it is necessary to separate the animations at intervals in turn. So the motions of all symbols have cycles that do not last too long. When the animation have a long period of time, it is imperative to alternate one another in the following way: the beginning of this motion at the time of the fuzzy recognition of the other motion that is taking place before it (Figure 8). (Tinghua Ai, 1998).
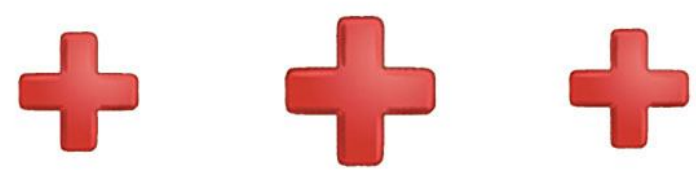

a. Scene 1

b. Scene 2

c. Scene 3

Figure 2. Dynamic symbol change size.
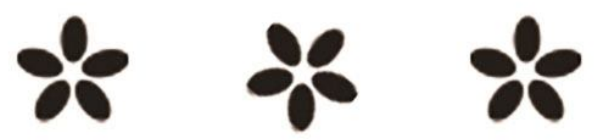

a. Scene 1

b. Scene 2

c. Scene 3
Figure 4. Dynamic symbol change direction.
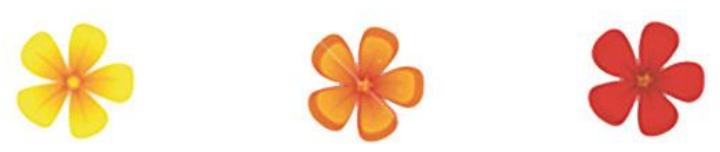
a. Scene 1
b. Scene 2
c. Scene 3

Figure 6. Dynamic symbol change colour.

\subsection{Basis and method of applying Wolfram Alpha online answering machine to create maps using dynamic symbols}

\subsubsection{Application basis}

Wolfram Alpha is an answering engine developed by Wolfram Research. This is an online service that responds directly to questions entered by calculating answers from structured data, not just providing a list of possible documents or websites contains answers the same way search engines do. This website was released to the public by Stephen Wolfram in 2009. Wolfram Alpha is written in 5 million lines of Mathematica code (using webMathematica and gridMathematica) and runs on 10,000 CPU (Guillermo Sánchez León José, 2017). 


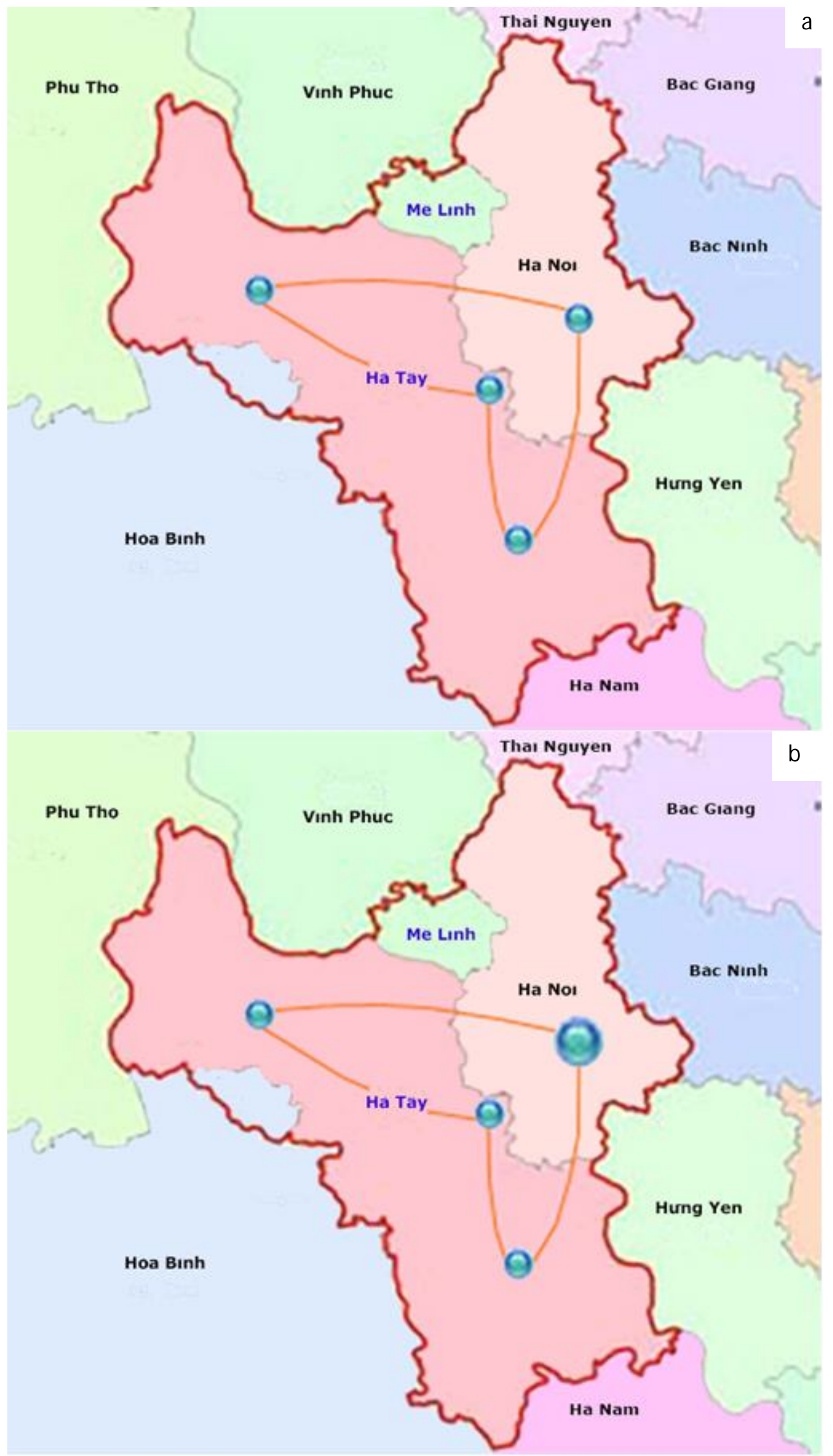




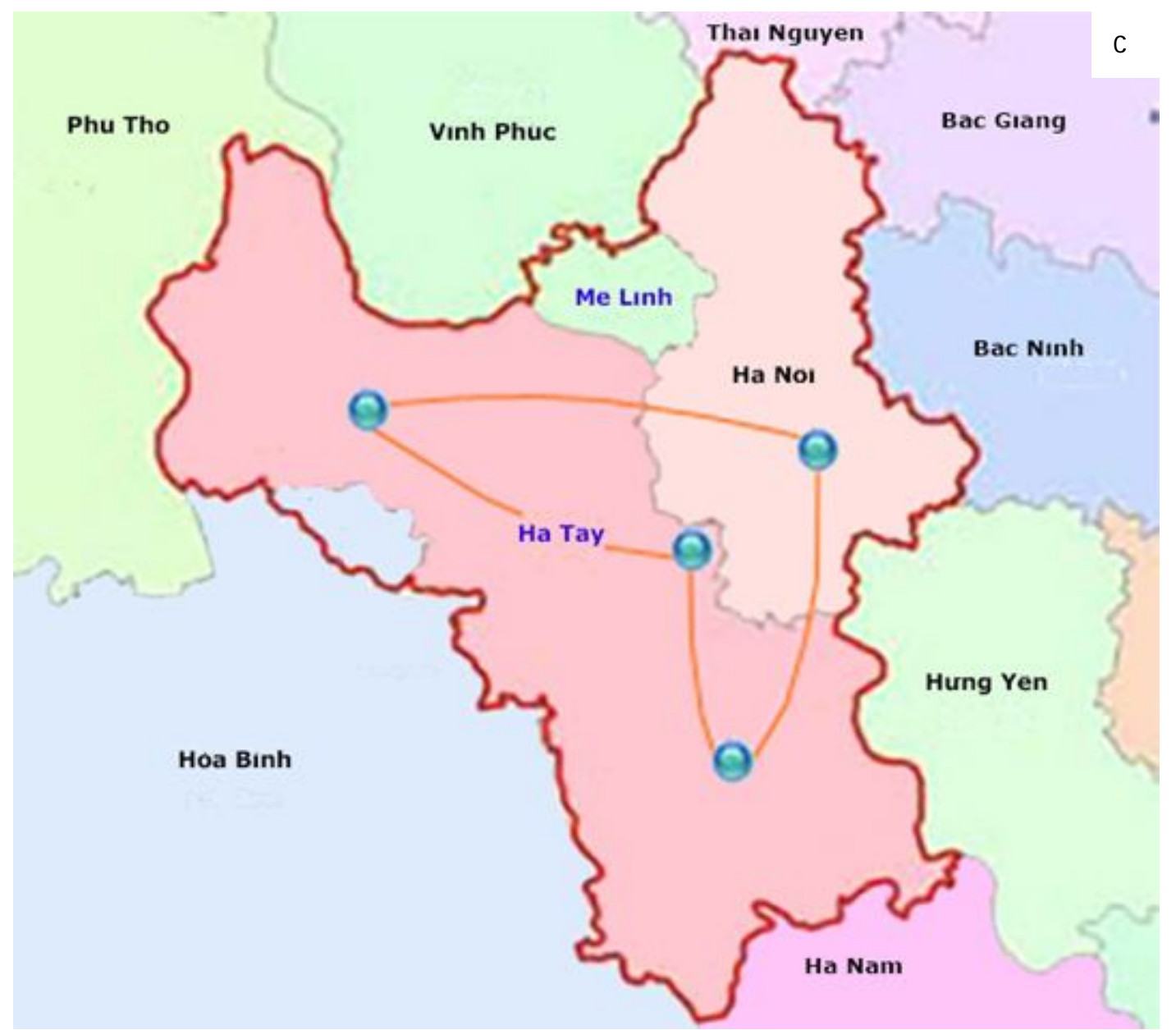

a. Scene 1: Dynamic symbol have the same form as static symbols;

b. Scene 2: Dynamic symbol are larger than static symbols;

c. Scene 3: Dynamic symbol returns to the same form as static symbols.

Figure 7. Dynamic symbol on the map change size while other symbols of the same type are static.

Animation is taking place

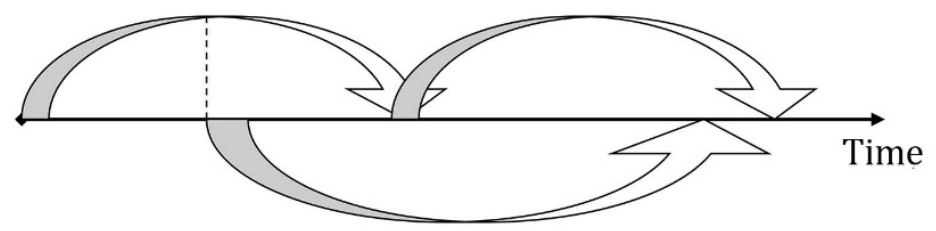

Alternate animation

Figure 8. The beginning of animation when all animations are alternating.

To perform single queries with a single sentence, the Wolfram Alpha website allows users to enter questions or operations directly into the search box. Wolfram Alpha's areas of computation and answers include math, science and technology, society and culture, everyday life. To perform complex calculations step by step and answer questions, users have to upgrade to Wolfram Alpha Pro. When creating an account in
Wolfram Alpha Pro, each user is provided with a separate Notebook to store its own data links, commands, files, documents. All resources put in for Wolfram Alpha's answering engine to analyze and calculate the answers are data that users post on the internet. 
2.2.2. Method of using Wolfram Alpha online answering machine to create maps using dynamic symbols

In order to create dynamic map, users will have to use the paid version. The dynamic symbol is the circular symbol representing the number of people infected with Covid-19 in an administrative territory unit. Size of the symbol corresponding to the number of people increased over time.

The area study is People's Republic of China. It is the first disease outbreak country in the world. People's Republic of China is close to Vietnam's border.

The statistics of infected patients are the official data provided by John Hopkin CSSV after the survey of WHO health experts who came to China during the recent outbreak. These statistics was downloaded in a file format. *.wl from Wolfram Alpha website and save at personal notebook of each user on the Wolfram Alpha website for commands with this file later.

Background map data and satellite images are provided by Wolfram Rearch. This data will be displayed when Wolfram Alpha user insert an online query with the device.

The procedure is as follows:
Step 1: Input data to draw circular symbols representing the number of patients. (Figure 9, Figure 10).

Step 2: Display the satellite background image which is provided by Wolfram Rearch. (Figure 11).

Step 3: Display the resize dynamic symbol showing the number of infected people increasing over time from the data file stored in Notebook format*.wl and labeling the number of infected people on the map. (Figure 12, Figure 13).

The size of a circular symbol is defined as the size of the circle bounding box. The diagonal length of the bounding box is specified in the mapping of the variable "date" in the data table. This mapping is determined by dividing linearly between the minimum set limits (smin) and the maximum (smax) (Guillermo Sánchez León José, 2017). Specifically here are as follows:

$\operatorname{smin}=0.01+$ minimum value of the variable "date" / 500

smax =maximum value of the variable "date"

The reason the smin value is set like this because if smin $=0.01$, the round symbol is not enough to show on the map, so the smin needs to be greater than the 0.01 value.

Step 4: Export the map as a gif file. (Figure 14, Figure 15).

$$
\begin{aligned}
& \ln [-]:=\text { updates =Union@Normal@dataset [All, "Last Update"]; } \\
& \ln [-]:=\text { regions[date_Dateobject] := Interpreter["AdministrativeDivision"][Normal@dataset[Select[\#["I } \\
& \text { \#["Country/Region"] == China countRY \&], "Province/State"]]; } \\
& \ln [-]:=\text { confirmations [date_Dateobject] := Normal@dataset }[\text { Select }[\#[\text { "Last Update"] == date \&\& \#["Country } \\
& \text { \&], "Confirmed"] /. Missing["Empty"] } \rightarrow \text { } \\
& \ln (-]:=\operatorname{get} \text { Association[date_DateObject] := AssociationThread[regions[date], confirmations[date] ] } \\
& \ln [-]=\text { getAssociation [ Mon } 27 \text { Jan 2020 20:30:00GMT-6. ] }
\end{aligned}
$$

Figure 9. The queries put into execution step 1.

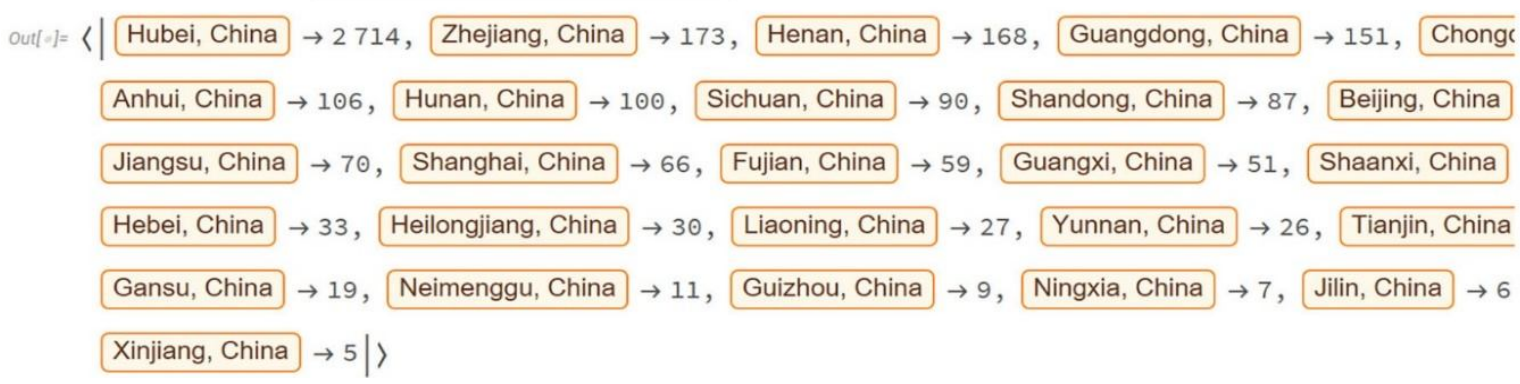

Figure 10. The machine answers the command in step 1. 


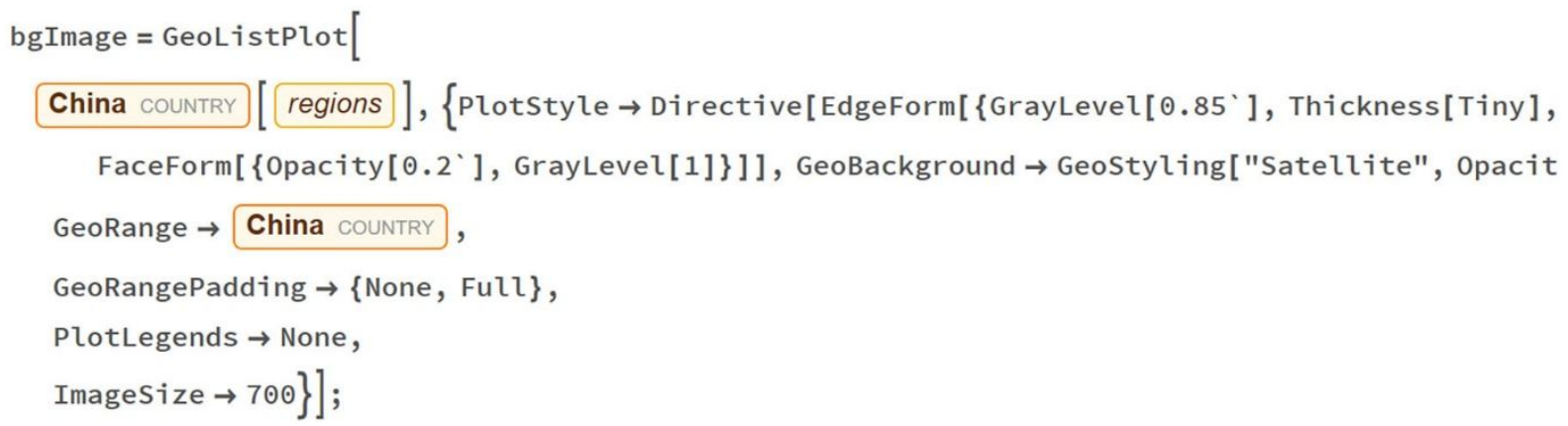

Figure 11. The queries put into execution step 2.

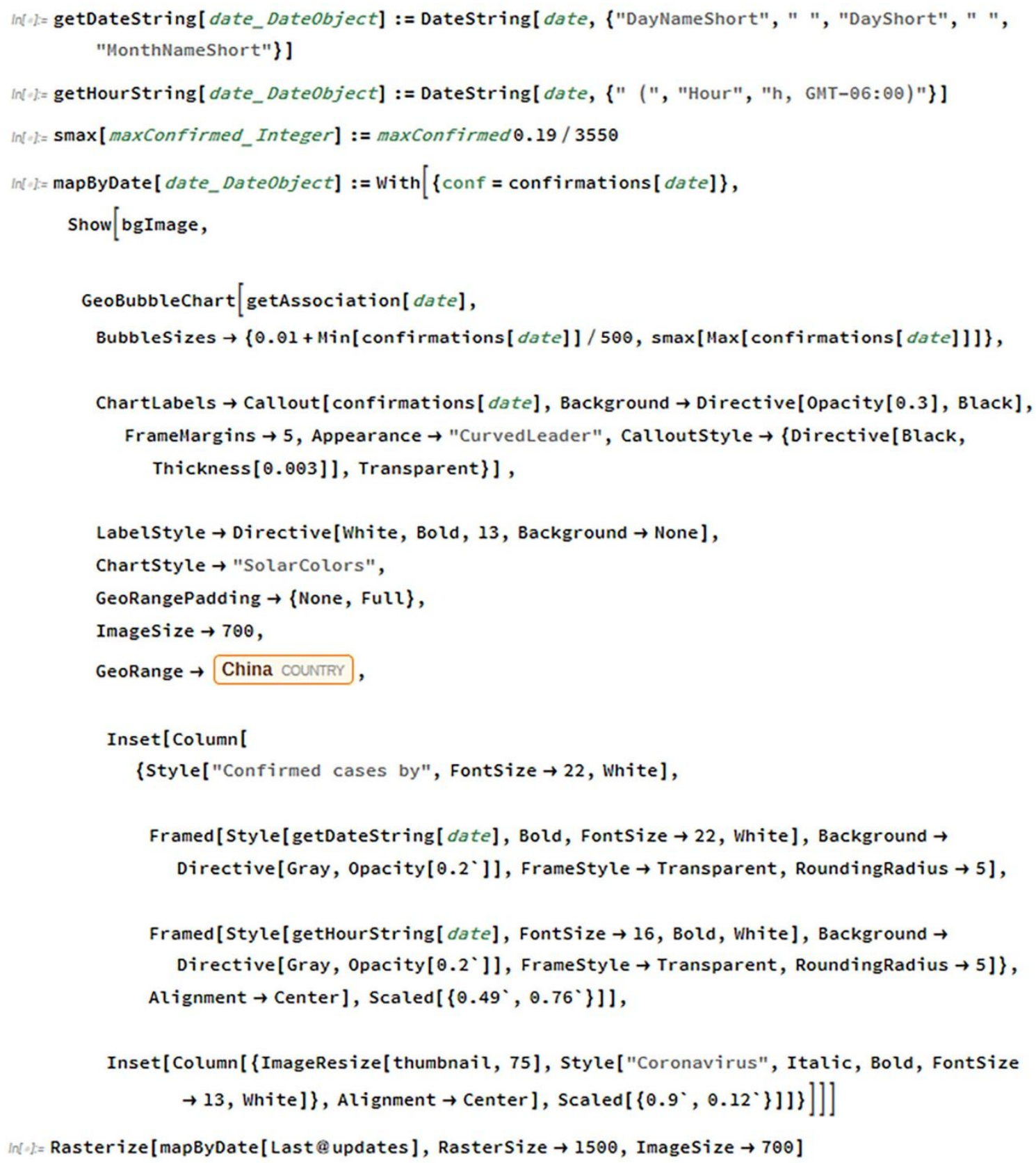

Figure 12. The queries put into execution step 3. 


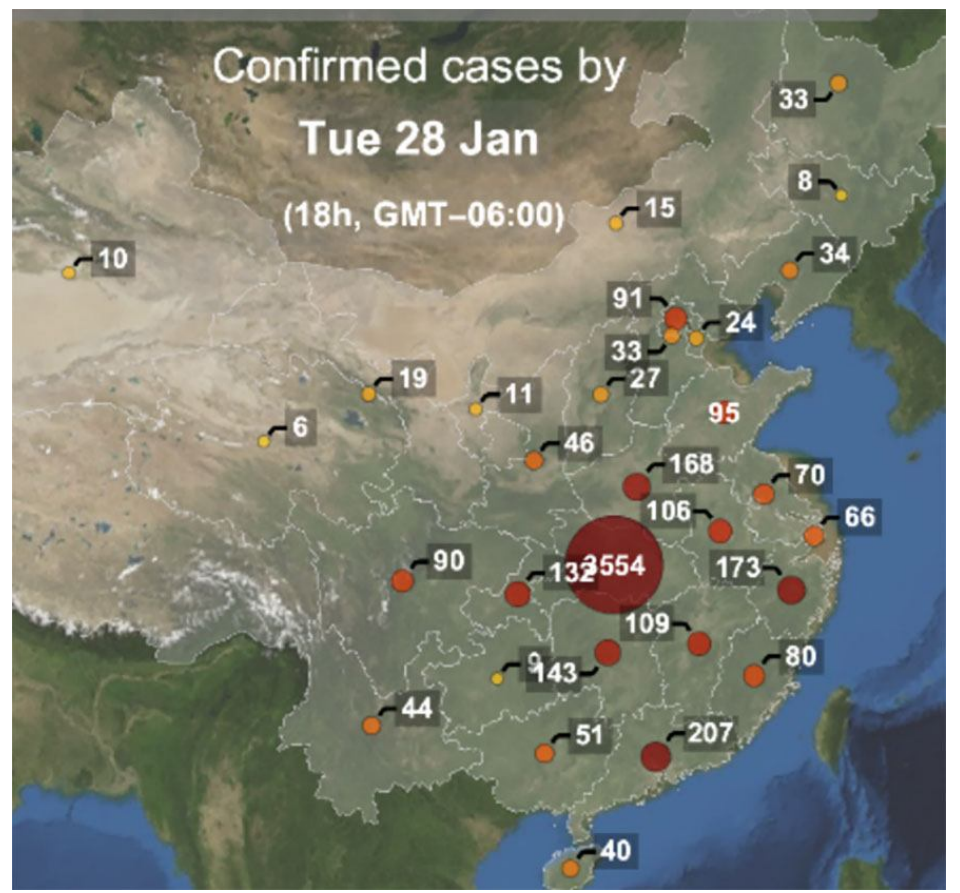

Figure 13. The queries put into execution step 3.

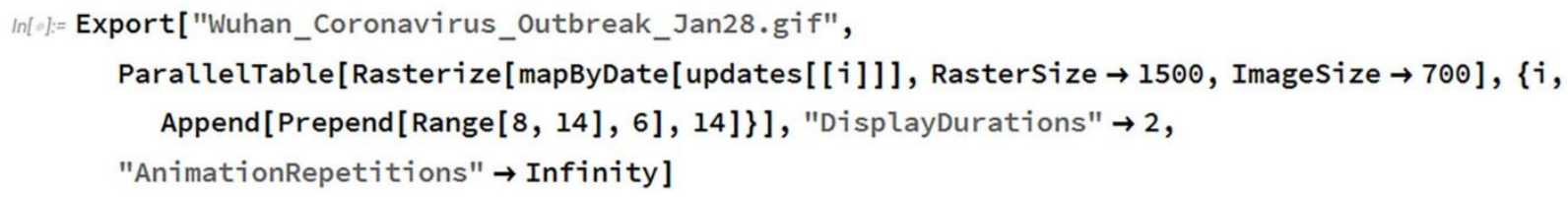

Figure 14. The queries put into execution step 4.

Out $[$ ol=Whan_Coronavirus_Outbreak_Jan28.gif

Figure 15. The machine answers the command in step 4.

\section{Result and discussion}

\subsection{Result}

The gif map contains 9 photos at 9 different times: $00 \mathrm{~h} 24^{\text {th }}$ January $2020,12 \mathrm{~h} 25^{\text {th }}$ January, $2020,11 \mathrm{~h} 26^{\text {th }}$ January 2020 , 09h 27th January $2020,19 \mathrm{~h} 27^{\text {th }}$ January $2020,13 \mathrm{~h}$ 28th January, $2020,18 \mathrm{~h} 28^{\text {th }}$ January 2020 , 23h $28^{\text {th }}$ January 2020 , and $13 \mathrm{~h} 29^{\text {th }}$ January 2020. In each time, the map shows the number of infected people by a circular symbol located in the center of each province's territory. The series of 9 maps running in the gif file will show the dynamic circular symbol that changes the size in proportion to the increased number of cases (Figure 16).

\subsection{Discussion}

Gif maps with dynamic symbols built on the basis of exploiting available resources on the Internet are quickly created, meeting the urgent need when creating thematic maps. In the final result shown above, the author of the article is not the one who made all steps to display for resources on the final gif map. The author of the paper only conducted research in a few stages in the process of asking for map resources in order to disseminate a direction for the creation of dynamic symbols. The ability to apply this direction to create dynamic symbols for Vietnam map is also possible, because all the commands to give answers from the data in *.wl file are connected in the above method section. But in 

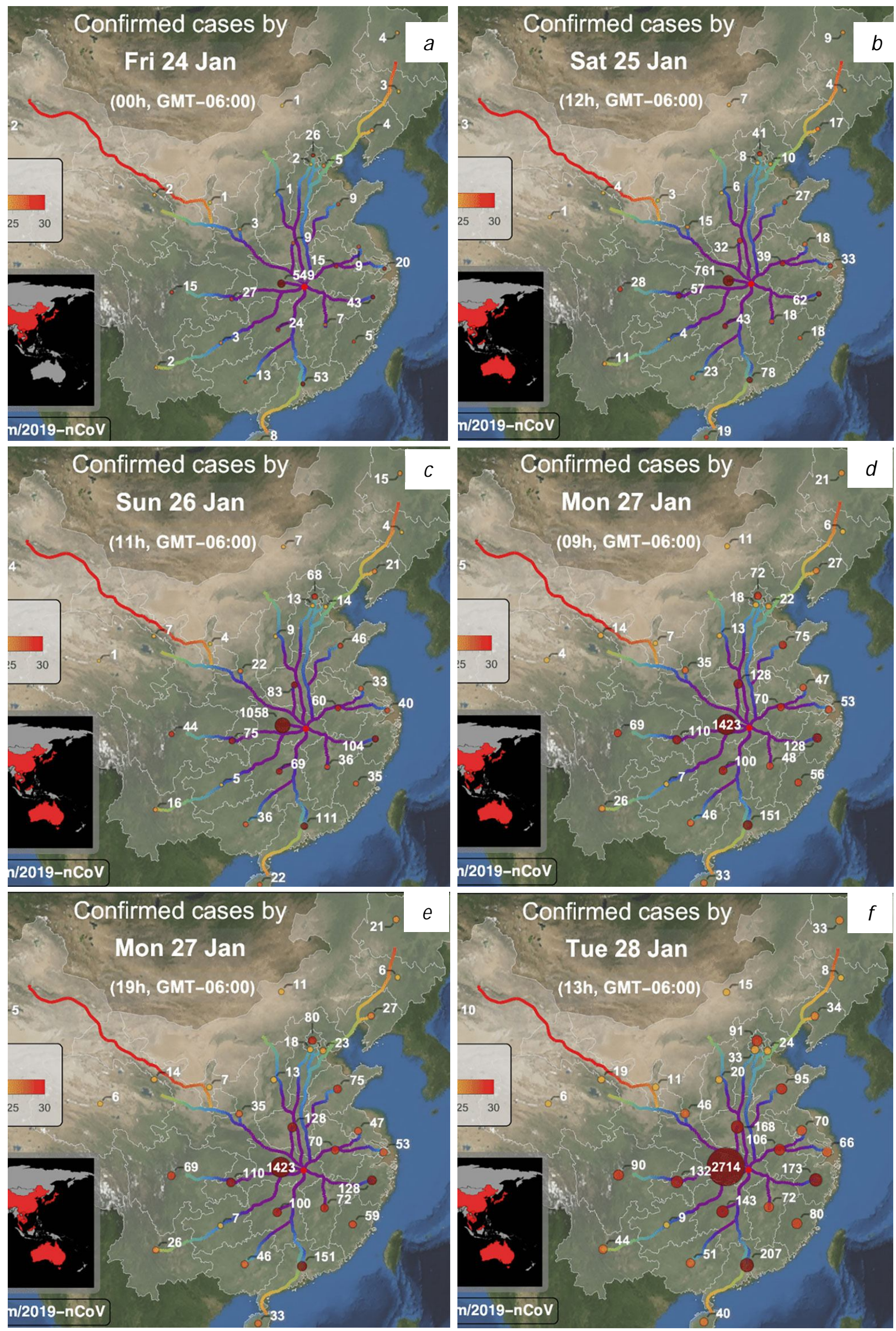

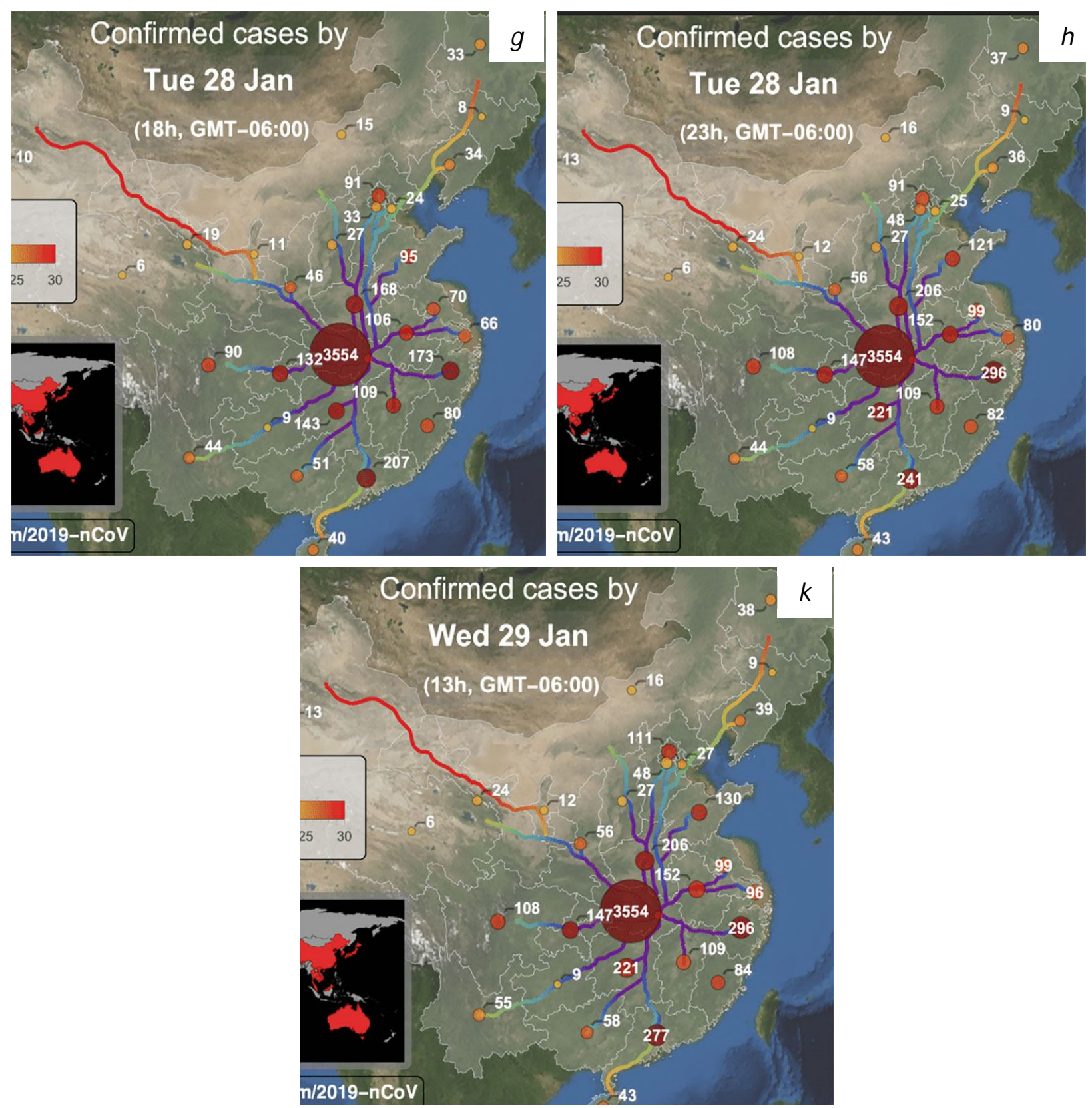

a. Scene 1: $00 \mathrm{~h}$ date 24/ 1; b. Scene 2: $12 \mathrm{~h}$ date 25/ 1; c. Scene 3: $11 \mathrm{~h}$ date 26/ 1; d. Scene 4: 09h date 27/ 1; e. Scene 5: 19h date 27/ 1; f. Scene 6: 13 h date 28/ 1; g. Scene 7: 18 h date 28/ 1; h. Scene 8: $23 \mathrm{~h}$ date $28 / 1 ; \mathrm{k}$. Scene 9: $13 \mathrm{~h}$ date 29/ 1 .

Figure 16. Time series images show an increase in the number of infected people through circular dynamic symbols.

Vietnam, the data is not updated in the data source of Wolfram Alpha so it has not been possible to link the order entry with the disease data in Vietnam. To do that, the raw data set needs to create and then use the SematicImport statement group to define the values in the raw data set as a *.wl file for machine to understand and execute.

\section{Conclusions}

Creating dynamic symbols is a good way to exploit the map presentation. The use of dynamic symbols contributes to the visualization and easy to recognition the contents of the symbols for most users with different levels of knowledge. That brings the map and the information on the 
map closer to everyone. With the implementation of the dynamic symbol map which this article introducts only requires effort, time, and reasonable cost, the desire of the author is that disseminating a simple method to apply the dynamic symbol more active in Vietnam.

\section{References}

Bui Ngoc Quy, Nguyen Phuc Chinh, Đong Duy Manh, Pham Van Hiep, Nguyen Thi My Hanh, Tran Trung Anh, Duong Anh Quan, Nguyen Thi Thu Ha, 2019. Remote navigation signal management and monitoring solfware development based on data colected from AIS and ENC, Journal of Mining and Earth Sciences Vol 60 Issue 4, 1-13.

DiBiase David, M. MacEachren Alan, B. Krygier John \& Reeves Catherine, 1992. Animation and the Role of Map Design in Scientific Visualization. Journal Cartography and Geographic Information Systems Vol 19 Issue 4, 201-214.

Fairbairn David \& Niroumand Jadidi Milad, 2013. Influential Visual Design Parameters on TV Weather Maps. The Cartographic Journal Vol 50 Issue 4, 311-323.

Guillermo Sánchez León José, 2017. Mathematica Beyond Mathematics: The Wolfram Language in the Real World. Chapman and Hall Book, New York, 462 pages.

Kim Quang Minh, 2019. Publishing and electronic publishing of the environmental resources industry today - practical needs and challenges, Journal of Mining and Earth Sciences Vol 60 Issue 4, 112-116.

Lai Poh Chin \& Yeh Anthony Gar On, 2004. Assessing the Effectiveness of Dynamic Symbols in Cartographic Communication. The Cartographic Journal Vol 41 Issue 3, 229-244.

Mai Van Sy, 2019. Virtual 3D city technology for urban planning and management in Kien Thuy - Hai Phong, Journal of Mining and Earth Sciences Vol 60 Issue 4, 14-21.

P. Peterson Michael, 1993. Interactive Cartographic Animation. Journal Cartography and Geographic Information Systems Vol 20 Issue 1, 40-44.

Paweł Cybulski, 2014. Rotating Point Symbols on Animated Maps for the Presentation of Quantitative Data. Journal of Cartography and Geographic Information Vol 64, 198-203.

Qian Cheng, Lei Zhou, Hong Cheng, 2013. A Model and Algorithm of Dynamic Map Expression and Its Application. Applied Mechanics and Materials, 321-324.

Tinghua Ai, 1998. Dynamic Symbol and Dynamic Map. Journal of Wuhan Technical University of Surveying and Mapping, 283.1-283.7.

Tran Trung Hong, 2001. Visualization Cartography. Transport Publishing House, Hanoi, 140 pages. 